\title{
АСИМПТОТИЧЕСКОЕ ОПИСАНИЕ ПРОЦЕССА РАСПРОСТРАНЕНИЯ ОДНОМЕРНОГО ИМПУЛЬСА В НЕОДНОРОДНОЙ СРЕДЕ
}

\author{
(Представил Н. Алумяэ)
}

Разнообразие неоднородных сред диктует необходимость разработки алгоритмов для описания волновых процессов в средах, свойства которых произвольным образом плавно меняются по пространственной координате. С математической точки зрения это приводит к необходимости решения уравнений математической физики с заданными переменными коэффициентами. Обычно такая задача решается лучевым методом $\left[{ }^{1-4}\right]$. Поскольку лучевой метод имеет ряд существенных недостатков [ $\left.{ }^{5}\right]$, целесообразно изучение исходных уравнений с помощью других аналитических методов $\left[{ }^{5-10}\right]$. В данной статье одномерная задача распространения волны в сильно неоднородной среде решается с помощью преобразования Лапласа и метода ВКБ. Аналогичный подход использован в $\left[{ }^{8-10}\right]$, где рассмотрены некоторые частные виды неоднородности среды при которых ВКБ-решение является точным $\left[{ }^{8}\right]$, и выведено решение для случая неоднородной среды с постоянной плотностью $\left[{ }^{9,10}\right]$. Ниже помимо сжимаемости среды переменной считается и ее плотность. Найдено решение, позволяющее следить за эволюцией формы волны в ее прифронтовой зоне. В частном случае решение сравнивается с точным аналитическим решением и обсуждается возможность использования его при решении задачи импульсной акустодиагностики свойств среды. 1. Одномерный волновой процесс в лагранжевой системе декартовых координат описывается уравнением

$$
U_{, x x}(x, t)+f_{1}(x) U_{, x}(x, t)-f_{2}(x) U_{, t t}(x, t)=0,
$$

где $x$ - лагранжева координата, $t$-время и $U(x, t)-$ перемещение материальных точек среды в направлении $x$.

В случае неоднородной упругой среды с переменной плотностью $Q_{0}(x)$ и сжимаемостью $\beta(x)$ коэффициенты уравнения (1.1) выражаются в виде

$$
\begin{aligned}
& f_{1}(x)=\beta_{, x}(x) \beta^{-1}(x), \\
& f_{2}(x)=\varrho_{0}(x) \beta^{-1}(x)>0 .
\end{aligned}
$$

Ограничимся случаем, когда функции $f_{1}(x)$ и $f_{2}(x)$ являются непрерывно дифференцируемыми в области $0 \leqslant x<\infty$.

Уравнение (1.1) решается при нулевых начальных условиях

$$
U(x, 0)=0, \quad U_{, t}(x, 0)=0
$$

и при краевом условии 


$$
U_{, t}(0, t)=\hat{c} \varphi(t) H(t),
$$

где $H(t)-$ функция Хевисайда, $\hat{c}-$ постоянное и $\varphi(t)-$ произвольная гладкая ограниченная функция.

Рассматривается волна, распространяющаяся в положительном направлении оси $x$ и предполагается малость деформации $U_{, x}(x, t) \ll 1$.

Поскольку коэффициенты уравнения (1.1) являются функциями только координаты $x$, при решении его используется преобразование Лапласа по времени. С учетом начальных условий (1.4) преобразованное уравнение движения среды принимает вид

$$
U_{, x x}^{L}(x ; p)+f_{1}(x) U_{, x}^{L}(x, p)-p^{2} f_{2}(x) U^{L}(x, p)=0 .
$$

Здесь $p$ - параметр преобразования Лапласа.

Уравнение (1.6) решается методом ВКБ. Подстановкой

$$
U^{L}(x, p)=\exp \left\{\int\left[p v(x, p)-\frac{1}{2} f_{1}(x)\right] d x\right\}
$$

оно сводится к уравнению Риккати

$$
p v, x(x, p)+p^{2} v^{2}(x, p)=p^{2} f_{2}(x)+g(x),
$$

где

$$
g(x)=\frac{1}{2}\left[f_{1, x}(x)+\frac{1}{2} f_{1}^{2}(x)\right] .
$$

Решение уравнения (1.8) ищется в виде ряда

$$
v(x, p)=\sum_{k=0}^{\infty} v_{k}(x) p^{-k} .
$$

После подстановки (1.9) в уравнение (1.8) и сравнения коэффициентов при одинаковых степенях $p$ решением уравнения (1.6) будет выражение

$$
\begin{aligned}
U^{L}(x, p) & =C_{1} \exp \left[-p \Phi_{2}(x)+\Phi_{1}(x)-p^{-1} \Phi_{3}(x)+O\left(p^{-2}\right)\right]+ \\
& +C_{2} \exp \left[p \Phi_{2}(x)+\Phi_{1}(x)+p^{-1} \Phi_{3}(x)+O\left(p^{-2}\right)\right],
\end{aligned}
$$

где

$$
\begin{aligned}
\Phi_{1}(x) & =-\frac{1}{4} \int_{0}^{x}\left[2 f_{1}(x)+f_{2, x}(x) f_{2}^{-1}(x)\right] d x, \\
\Phi_{2}(x) & =\int_{0}^{x} f_{2}^{1 / 2}(x) d x, \\
\Phi_{3}(x) & =\frac{1}{2} \int_{0}^{x} f_{2}^{-1 / 2}(x)\left[g(x)+\frac{1}{4} f_{2, x x}(x) f_{2}^{-1}(x)-\right. \\
& \left.-\frac{5}{16} f_{2, x}^{2}(x) f_{2}^{-2}(x)\right] d x .
\end{aligned}
$$

Учитывая условие (1.5), получим из (1.10) выражение $U^{L}(x, p)=\hat{c} p^{-1} \varphi^{L}(p) \exp \left[-p \Phi_{2}(x)+\Phi_{1}(x)-p^{-1} \Phi_{3}(x)+O\left(p^{-2}\right)\right]$

Для обращения (1.14) перепишем его в форме 


$$
\begin{aligned}
U^{\dot{L}}(x, p) & =\hat{c} p^{-1} \varphi^{\dot{L}}(p) \exp \left[-p \Phi_{2}(x)+\Phi_{1}(x)+p^{-1} \Phi_{3}(x)\right] \times \\
& \times \exp \left[p^{-2} \Phi_{4}(x)+p^{-3} \Phi_{5}(x)+\ldots+p^{-n} \Phi_{n+2}(x)+\ldots\right]
\end{aligned}
$$

и используем разложение

$$
\begin{array}{r}
\exp \left[p^{-2} \Phi_{4}(x)+p^{-3} \Phi_{5}(x)+\ldots+p^{-n} \Phi_{n+2}(x)+\ldots\right] \sim \\
\sim 1+p^{-2}\left\{\Phi_{4}(x)+p^{-1} \Phi_{5}(x)+p^{-2}\left[\Phi_{6}(x)+\frac{1}{2} \Phi_{4}^{2}(x)\right]+O\left(p^{-3}\right)\right\} .
\end{array}
$$

В результате получим решение исходного уравнения (1.1) в виде

$$
\begin{aligned}
U(x, t) & =\hat{c} H(\zeta) \exp \Phi_{1}(x)\left\{\int_{0}^{\zeta} \varphi(\zeta-\xi) F_{0}(\eta) d \xi+\right. \\
& +\Phi_{4}(x) \int_{0}^{\zeta} \vartheta(x, \xi) \varphi(\zeta-\xi) F_{2}(\eta) d \xi+ \\
& \left.+\Phi_{5}(x) \int_{0}^{\zeta} \vartheta^{3 / 2}(x, \xi) \varphi(\xi-\xi) F_{3}(\eta) d \xi+\ldots\right\}
\end{aligned}
$$

где

$$
\begin{gathered}
\eta=2\left[\xi\left|\Phi_{3}(x)\right|\right]^{1 / 2}, \quad \vartheta(x, \xi)=\xi /\left|\Phi_{3}(x)\right|, \\
F_{n}(\eta)=\left\{\begin{array}{lll}
J_{n}(\eta), & \text { при } & \Phi_{3}(x) \geqslant 0 \\
I_{n}(\eta), & \text { при } & \Phi_{3}(x) \leqslant 0, \\
\zeta(x, t)=t-\Phi_{2}(x) .
\end{array}\right.
\end{gathered}
$$

Здесь $J_{n}(\eta)$ и $I_{n}(\eta), n=0,1,2, \ldots$ - функции Бесселя первого рода вещественного и мнимого аргументов соответственно.

При выводе решения (1.17) был использован ряд (1.9), сходящийся при больших значениях параметра $p$, и следовательно, решение (1.17) описывает волновой процесс в прифронтовой зоне волны, где выполняется условие

$$
\left|\zeta \Phi_{3}(x)\right| \ll 1 .
$$

2. В случае ограниченных и достаточно гладких функций $\Phi_{j}(x) \quad(j=$ $=4,5,6, \ldots)$ эволюцию формы волны в ее прифронтовой зоне можно приближенно описать первым членом решения (1.17)

$$
U(x, t)=\hat{c} H(\zeta) \exp \Phi_{1}(x) \int_{0}^{\zeta} \varphi(\zeta-\xi) F_{0}(\eta) d \xi,
$$

ибо при $\left|\zeta \Phi_{3}(x)\right| \rightarrow 0$ имеем $F_{0}(\eta) \rightarrow 1$ и $F_{i}(\eta) \rightarrow 0$ при $i>1$. Требуемое для этого выполнение условия (1.20) проиллюстрируем сравнением результатов расчета по выражению (2.1) и по точному решению, выведенному в $\left[{ }^{6}\right]$ для случая изменения свойств среды согласно формулам

$$
\beta(x)=\beta_{0}(1+\alpha x)^{m}, \quad \varrho_{0}(x)=\varrho_{0}(1+\alpha x)^{q}, \quad m=q+2 .
$$

Здесь $\beta_{0}, \varrho_{0}$ и $\alpha-$ постоянные.

При начальных и краевых условиях (1.4) и (1.5) точное решение примет вид

$$
\begin{gathered}
U(x ; t)=\hat{c}(1+\alpha x)^{(1-m) / 2} H[t-B(x)]\{P[t-B(x)]- \\
\left.-B(x) f \int_{0}^{t}\left[\tau^{2}-B^{2}(x)\right]^{-1 / 2} P(t-\tau) J_{1}\left[\left(\tau^{2}-B(x)\right)^{1 / 2} f\right] d \tau\right\},
\end{gathered}
$$


где

$$
\begin{aligned}
& P(t)=\int_{0}^{t} \varphi(\tau) d \tau, \quad c_{0}^{2}=\varrho_{0}^{-1} \beta_{0}, \quad b=\left(\alpha c_{0}\right)^{-2}, \\
& f=\frac{1}{2}(1-m) b^{-1 / 2}, \quad B(x)=b^{1 / 2} \ln (1+\alpha x) .
\end{aligned}
$$

Рассмотрим волну, возбужденную краевым воздействием

$$
U_{, t}(0, t)=\hat{c} H(t) \sin \omega t,
$$

где $\omega-$ угловая частота возмущения.

Результаты сравнения при значениях параметров $m=2, \alpha=1 \mathrm{~m}^{-1}$, $c_{0}=3 \cdot 10^{3}$ мс $^{-1}$ и расстояний $x=1$ м представлены на рис. 1. Здесь $T$ и $U_{0}$ - период и удвоенная амплитуда краевого воздействия; введено обозначение $x=\left|\xi \Phi_{3}(x)\right|$.

Из рис. 1 видно, что формы двух первых периодов распространяющейся в среде волны, вычисленные по асимптотическому решению (2.1) при значении величины $x=10^{-2}$ и по точному решению (2.3) при $x=10^{-1}$, если $\zeta T^{-1}=2$ (кривая 3 ), практически совпадают, а результат расчета по формуле (2.1) при $\chi=10^{-1}$ (кривая 2) качественно правильно описывает форму волны. Результаты расчета при значении величины $x$ равной 10 по точному решению (кривая 4) и асимптотическому решению (2.1) (кривая 1) расходятся качественно. Следовательно, хорошее совпадение сравнимых результатов наблюдается при $x<10^{-1}$, и число $n$ правильно описываемых периодов волны за фронтом определяется выражением

$$
n<\left[10^{2} \pi\left|\Phi_{3}(x)\right|\right]^{-1} \omega .
$$

Здесь необходимо отметить, что с результатами расчета, представленными кривой 3 , совпадает результат расчета по формуле (2.1) при $F_{0}(\eta)=1$, т. е. при пренебрежении вклада функции Бесселя в решении.

Существенным является также тот факт, что главная часть изменения амплитуды в решении (2.3) точно равняется аналогичной части в асимптотическом решении (2.1).

Из сопоставления результатов расчета следует, что при выполнении условия (1.20) первое асимптотическое приближение решения (2.1) с достаточной для практики точностью описывает эволюцию формы волны в неоднородной среде.

Далее на основе $(1.11),(1.12),(1.13)$ с учетом выражений (1.2) и (1.3) определяем функции $\Phi_{1}(x), \Phi_{2}(x)$ и $\Phi_{3}(x)$ через сжимаемость $\beta(x)$ и плотность $\varrho_{0}(x)$ среды

$$
\begin{aligned}
\Phi_{1}(x) & =-\frac{1}{4} \int_{0}^{x}\left[\beta, x(x) \beta^{-1}(x)+\varrho_{0, x}(x) \varrho_{0}^{-1}(x)\right] d x, \\
\Phi_{2}(x) & =\int_{0}^{x}\left[\varrho_{0}(x) \beta^{-1}(x)\right]^{1 / 2} d x, \\
\Phi_{3}(x) & =\frac{1}{32} \int_{0}^{x}\left[\beta(x) \varrho_{0}^{-1}(x)\right]^{1 / 2}\left\{4 \left[\beta, x x(x) \beta^{-1}(x)+\right.\right. \\
& \left.+\varrho_{0, x x}(x) \varrho_{0}^{-1}(x)-\varrho_{0, x}^{2}(x) \varrho_{0}^{-2}(x)\right]- \\
& \left.-\left[\beta, x(x) \beta^{-1}(x)-\varrho_{0, x}(x) \varrho_{0}^{-1}(x)\right]^{2}\right\} d x .
\end{aligned}
$$




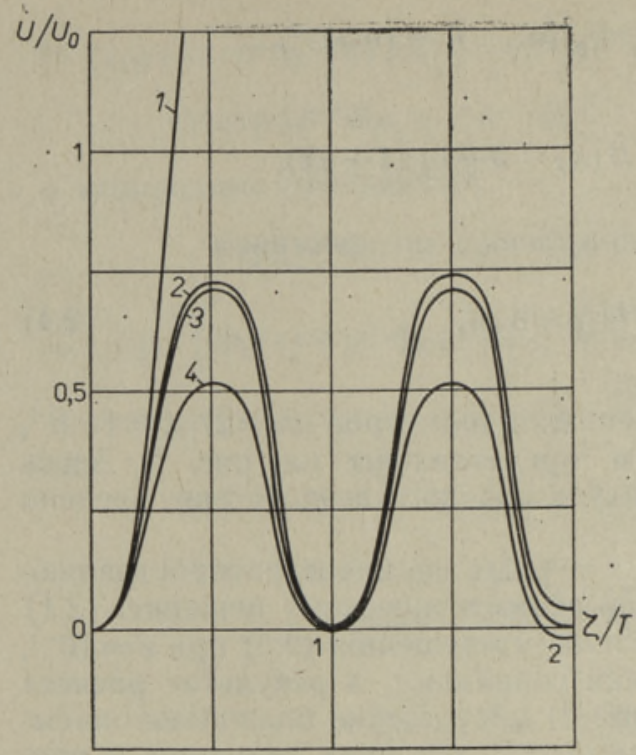

Рис. 1.

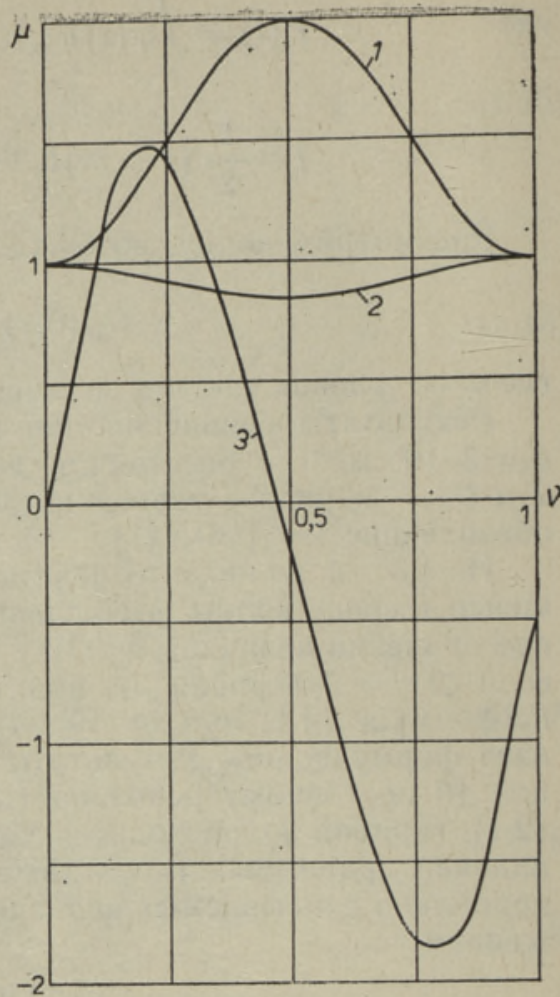

Рис. 2.

Интеграл в выражении (2.5) удается вычислить, и главная часть амплитуды волны определяется выражением

$$
\exp \Phi_{1}(x)=\left[\beta(0) \varrho_{0}(0) \beta^{-1}(x) \varrho_{0}^{-1}(x)\right]^{1 / 4} .
$$

Выражение (2.6) для функции $\Phi_{2}(x)$ точно определяет время распространения фронта волны в неоднородной среде и совпадает с аналогичными выражениями, полученными другими методами $[3,11]$.

Интересно отметить, что в случае однородной среды решение (2.1) преобразуется к виду

$$
U(x, t)=\hat{c} H(\zeta) \int_{0}^{\zeta} \varphi(\zeta-\xi) d \xi
$$

и является точным.

В качестве примера рассмотрим распространение волны в среде, свойства которой меняются по формулам

$$
\beta(x)=\beta_{0}+\beta_{1}\left[1-\cos \left(2 \pi x / c_{0} T\right)\right], \quad \varrho_{0}(x)=\varrho_{0},
$$

где $\beta_{0}, \beta_{1}, \varrho_{0}-$ постоянные.

Пусть краевое воздействие задано выражением (2.4). Вычисления проведены на основе решения (2.1) при значениях параметров $c_{0}=$ $=5 \cdot 10^{3} \mathrm{Mc}^{-1}$ и $\beta_{1} \beta_{0}{ }^{-1}=0,5$.

На рис. 2 кривой $1\left(\mu=\beta / \beta_{0}, \nu=x / c_{0} T\right)$ изображена функция изменения сжимаемости среды $(2.10)$ и кривой $3\left(\mu=10^{-3} \Phi_{3}(x) c^{-1}\right)$ показано соответствующее изменение характерной функции $\Phi_{3}(x)$. Получается, что амплитуды двух первых периодов волны при выполнении условия $x \ll 10^{-2}$ при $\zeta T^{-1}=1$ изменяются согласно кривой $2\left(\mu=U / U_{0}\right)$.

Представляет интерес проследить за расчетной формой волны в зави- 


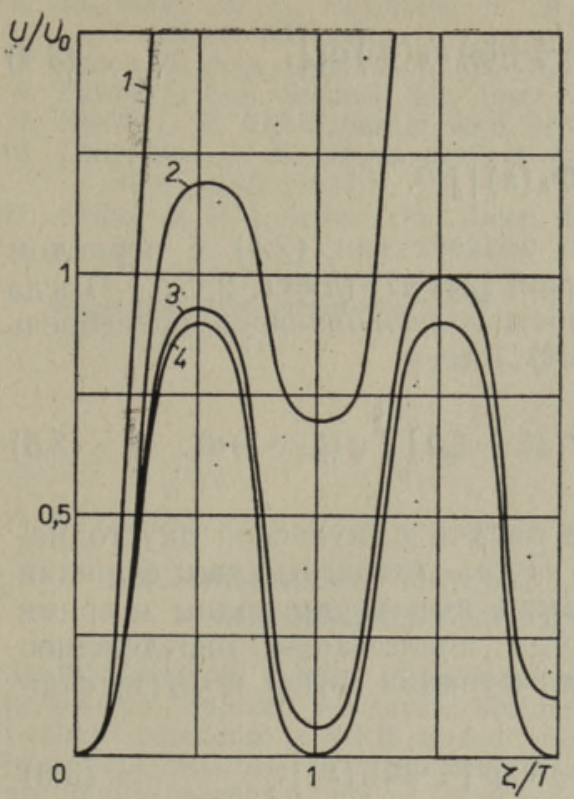

Рис. 3.

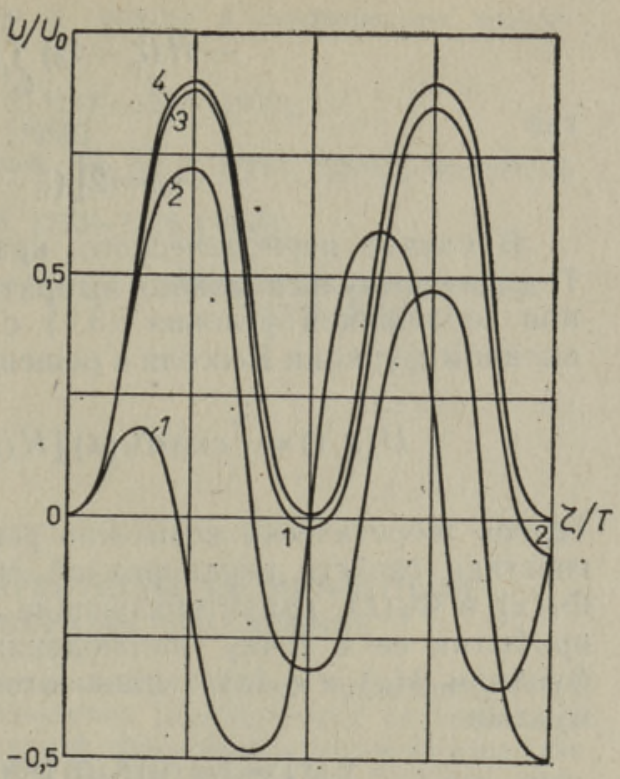

Рис. 4.

симости от строгости выполнения условия (1.20). На рис. 3 и 4 приведены результаты расчета формы двух первых периодов волны при $v=0,25$ и $v=0,75$, т. е. при $\Phi_{3}(x)>0$ и $\Phi_{3}(x)<0$ соответственно. Получается, что в обоих случаях результаты расчета при $x \leqslant 10^{-2}$ представимы кривой 4 и в случае $x=10^{-1}$ при $\zeta T^{-1}=1$ они еще качественно правильно описывают форму волны (кривая 3). Кривые 1 и 2, вычисленные при значениях величины $x$ равной 10 и 1 соответственно при $\zeta T^{-1}=1$, свидетельствуют о неприменимости решения (2.1) в этих случаях. Отметим также, что при $\Phi_{3}(x)>0$ с уменьшением значения величины $x$ решение приближается к кривой 4 со стороны бо́льших значений $U / U_{0}$ (рис. 3 ), а при $\Phi_{3}(x)<0$ со стороны меньших значений (рис. 4).

3. Поскольку решение (1.17) применимо в прифронтовой зоне волны, то оправдано использование его при описании распространения конечных коротких импульсов в неоднородной среде. Длина импульса ६о должна при этом удовлетворять условию

$$
\left|\zeta_{0} \Phi_{3}(x)\right| \ll 1
$$

Решение уравнения (1.1) при импульсном краевом воздействии

$$
U_{, t}(0, t)=\hat{c} \varphi(t)\left[H(t)-H\left(t-\zeta_{0}\right)\right]
$$

и при начальных условиях (1.4) находится с учетом линейности уравнения (1.1). Путем простого сдвига начала отсчета времени $t$ на величину $\zeta_{0}$ сначала находится решение для случая краевого воздействия

$$
U_{, t}(0, t)=\hat{c}_{\varphi}(t) H\left(t-\zeta_{0}\right) .
$$

Вычитая полученное решение из (2.1), искомое решение при учете только первого асимптотического члена выражается формулой

$$
U(x, t)=\hat{c} \exp \Phi_{1}(x)\left[H(\zeta) \int_{0}^{\zeta} \varphi(\zeta-\xi) F_{0}\left(\eta_{0}\right) d \xi-\right.
$$




$$
\left.-H\left(\zeta-\zeta_{0}\right) \int_{\zeta_{0}}^{\zeta} \varphi\left(\zeta-\xi+\zeta_{0}\right) F_{0}\left(\eta_{0}\right) d \xi\right]
$$

где

$$
\eta_{0}=2\left[\left(\xi-\zeta_{0}\right)\left|\Phi_{3}(x)\right|\right]^{1 / 2} .
$$

В случае периодического краевого воздействия (2.4) с периодом $T$ длину импульса можно выбрать равной $\zeta_{0}=n T(n=1,2, \ldots)$. Тогда при соблюдении условия $(3.1)$ с точностью, позволяющей пренебречь вкладом функции Бесселя в решении (3.4), имеем

$$
U(x, t)=\hat{c} \exp \Phi_{1}(x)\left[H(\zeta)-H\left(\zeta-\zeta_{0}\right)\right] \int_{0}^{\zeta} \varphi(\zeta-\xi) d \xi
$$

Теперь теоретически возможно решение задачи импульсной акустодиагностики свойств неоднородной среды. Пусть известны две функции $\Phi_{1}(x)$ и $\Phi_{2}(x)$, характеризующие изменение амплитуды волны и время прибытия ее в точку наблюдения. Тогда подлежащие определению функции $\beta(x)$ и $\varrho_{0}(x)$ выражаются через функции $\Phi_{1}(x)$ и $\Phi_{2}(x)$ формулами

$$
\begin{gathered}
\beta(x)=\left[\varrho_{0}(0) \beta(0)\right]^{1 / 2} \Phi_{2, x}^{-1}(x) \exp \left[-2 \Phi_{1}(x)\right], \\
\varrho_{0}(x)=\beta(x) \Phi_{2, x}^{2}(x) .
\end{gathered}
$$

В случае измерения функций $\Phi_{1}(x)$ и $\Phi_{2}(x)$ при одном значении расстояния $x$ становится возможным определение двух неизвестных параметров, входящих в известные аналитические представления сжимаемости $\beta(x)$ и плотности $\varrho_{0}(x)$ среды.

Например, в простейшем случае, когда известно, что плотность среды $\varrho_{0}$ постоянная, а сжимаемость ее меняется линейно по формуле

$$
\beta(x)=\beta_{0}(1+\alpha x),
$$

и необходимо определить параметры $\alpha$ и $\beta_{0}$, то на основе формул (2.6) и $(2.8)$ имеем

$$
\begin{aligned}
& \Phi_{1}(x)=-\frac{1}{4} \ln (1+\alpha x), \\
& \Phi_{2}(x)=2 \alpha^{-1}\left(\varrho_{0} \beta_{0}^{-1}\right)^{1 / 2}\left[(1+\alpha x)^{1 / 2}-1\right] .
\end{aligned}
$$

Пусть измерения проводятся при $x=x_{*}$. Искомые параметры $\alpha$ и $\beta_{0}$ определяются тогда из (3.9) и (3.10) по формулам

$$
\begin{aligned}
& \alpha=x_{*}^{-1}\left\{\exp \left[-4 \Phi_{1}\left(x_{*}\right)\right]-1\right\}, \\
& \beta_{0}=4 \varrho_{0} x_{*}^{2} \Phi_{2}^{-2}\left(x_{*}\right)\left\{\exp \left[-2 \Phi_{1}\left(x_{*}\right)\right]+1\right\}^{-2} .
\end{aligned}
$$

Аналогично решается задача при постоянном $\beta$ и линейно меняющемся $\varrho_{0}(x)$.

\section{ЛИТЕРАТУРА}

1. Бабич В. М. ПММ, 25, вып. 1, 38-48 (1961).

2. Tokuoka, T., Shimanchi, K. J. Acoust. Soc. Amer., 73, № 2, 441-449 (1983).

3. Cooper, H. F. SIAM Rev., 9, № 4, 671-679 (1967).

4. Энгельбрехт Ю. К. Изв. ӒН СССР. Мех. тверд. тела, № 4, 74-81 (1979).

5. Никольский Э. В. Метод эквнвалентных систем в теории распространения волн. Новосибирск, «Наука», 1982. 
6. Филиппов Н. Г., Бахрамов Б. М. Волны в упругих и неоднородных средах. Ташкент, ФАН, 1978.

7. Eason, G. Acta mech., № 7, 137-160 (1969).

8. Eason, G. Bull. Seismol. Soc. Amer., 57, № 6, 1267-1277 (1967).

9. Steele, C. R. AIAA Journal, № 5, 896-902 (1969).

10. Longscope, D. B., Steele, C. R. J. Appl. Mech., 41, № 4, Trans. ASME, 96, Ser. E, 1057-1062 (1974).

11. Reddy, D. P. J. Acoust. Soc. Amer., 45, № 5, 1273-1276 (1969).
Институт кибернетики
Академии наук Эстонской ССР
Поступила в редакцию 23/V 1984

\section{A. RAVASOO}

\section{UHEMOOTMELISE IMPULSI LEVIKUPROTSESSI ASUMPTOOTILINE KIRJELDAMINE MITTEHOMOGEENSES KESKKONNAS}

Analüütiliselt on uuritud ühemōōtmelise pikilaine levikut tugevalt mittehomogeenses keskkonnas. Sujuvalt muutuvate omadustega keskkonna liikumisvõrrand on lahendatud Laplace'i teisenduse ja WKB meetodi abil. On saadud asümptootiline lahend kirjeldamaks laine kuju evolutsiooni frondilähedases piirkonnas. Saadud lahendit on võrreldud täpse analüütilise lahendiga, mis on leitud keskkonna omaduste muutuse ühel erijuhul. On vaadeldud võimalust lahendi kasutamiseks keskkonna omaduste impulss-akustodiagnostikas ja esitatud lahendi kehtivustingimus.

\section{A. RAVASOO \\ DESCRIPTION OF THE ONE-DIMENSIONAL PULSE PROPAGATION IN AN INHOMOGENEOUS MEDIUM}

The one-dimensional longitudinal wave propagation in the strongly inhomogeneous medium is investigated analytically. The equation of motion with smoothly variable properties of the medium taken into acount is solved, using the Laplace transform and the method of .WKB. The asymptotic solution describing the evolution of the wave-form in its near-front region is obtained. In a special case of inhomogeneity, when it is possible to find the exact analytical solution, the comparison of exact and asymptotical results is presented. The possibility of using the asymptotic solution for acoustic pulsediagnostics of properties of media is discussed, and the validity criterion of asymptotic solution is presented. 